\title{
Análisis y comparación de la norma contable local, americana e internacional en el tratamiento de activos derivados
}

\author{
José Arias Moya ${ }^{1}$ \\ Verena Yáñez Andrades ${ }^{2}$
}

Recibido: 17 agosto de 2015

Aprobado: 15 de noviembre de 2015

\begin{abstract}
Moya, J. \& Yáñez, V. (2015). Análisis y comparación de la norma contable local, americana e internacional en el tratamiento de activos derivados. Activos, 25, 107-134.
\end{abstract}

Clasificación JEL: G38, G39.

\section{Resumen}

El inminente desarrollo del mercado de derivados a nivel internacional, como así mismo en el nuestro (volumen promedio de transacciones cercano a USD 2.000 millones, lo que representa un $43 \%$ del volumen del mercado de contado) y el creciente interés de las empresas por cubrir sus riesgos financieros, motivó el desarrollo del presente documento en el cual se busca profundizar sobre el tratamiento contable de los activos derivados

1 José Tomás Arias Moya, Departamento de Economía y Finanzas, Facultad de Ciencias Económicas y Administrativas, Universidad Católica de la Santísima Concepción, Chile. Correo: josearias@ucsc.cl

2 Verena Alejandra Yáñez Andrades, Departamento de Auditoría y Sistemas de Información, Facultad de Ciencias Económicas y Administrativas, Universidad Católica de la Santísima Concepción, Chile. Correo: vyanez@ucsc.cl 
bajo las distintas normativas, enfocándonos básicamente en la clasificación, valoración, reconocimiento inicial y posterior de estos activos.

Este documento analiza y compara el tratamiento contable y financiero de los activos derivados bajo distintas normas contables, entre ellas: la norma local chilena según los PCGA ${ }^{3}$, la norma americana, bajo los USGAAP y la norma europea basada en IFRS, con el objetivo de determinar las diferencias significativas en el tratamiento contable de los activos derivados. Para lograr este objetivo, se procedió a la revisión bibliográfica de textos relacionados con el tema de valoración de activos derivados, administración del riesgo y estrategia de cobertura, así como el análisis de textos actualizados relacionados a las normas descritas, obteniendo como resultados preliminares algunas similitudes, como aquella en la cual todos los productos derivados deben reconocerse como activos y pasivos, en el balance de situación financiera, inicialmente a su valor razonable y algunas diferencias como las asociadas a los requerimientos formales en la contabilidad de coberturas, la revalorización de los activos derivados implícitos, los límites de las coberturas, entre otras.

\title{
Palabras clave
}

Contabilidad, activos derivados, finanzas.

Moya, J. \& Yáñez, V. (2015). Analysis and comparison of local, American and international Accounting regulation in the treatment of derivative assets. Activos, 25, 107-134.

\begin{abstract}
The imminent development of the derivatives market internationally, as likewise in ours (average transaction volume of around U.S. \$2,000 million, representing $43 \%$ of the cash market volume) and the growing interest of
\end{abstract}

3 Antes de la adopción de la normativa contable internacional. 
companies to cover financial risks, motivated us to deepen on the accounting treatment of these assets under the various regulations, focusing primarily on the classification, assessment, initial and subsequent recognition of these assets. Therefore, we have developed a project that analyzes and compares the accounting and financial derivative assets under different accounting rules, including: our local standard according to $\mathrm{GAP}^{4}$, American standard, under U.S. GAAP and European Standard based on IFRS, in order to determine significant differences in the accounting treatment of derivative assets given the application of the above standards. To do this, we proceeded to the literature review of texts related to the topic of derivative asset valuation, risk management and hedging strategy and analysis updated texts related to the standards described, obtaining preliminary results, some similarities one that is related to all derivatives be recognized as assets and liabilities in the statement of financial position, initially at fair value and some differences as those associated with the formal requirements for hedge accounting, the asset revaluation embedded derivatives, the limits of the coverage, among others.

\section{Keywords}

Accounting, derivative assets, finance.

\section{Moya, J. \& Yáñez, V. (2015). Analyse et comparaison de la norme comptable locale, américaine et internationale dans le traitement d’actifs dérivés. Activos, 25, 107-134.}

\section{Résumé}

Le développement imminent du marché des produits dérivés à léchelle internationale, comme aussi dans le nôtre (volume moyen de près de 2.000 millions d'USD de transactions, ce qui représente $43 \%$ du volume du marché comptable) et l'intérêt croissant des entreprises pour couvrir

4 Before the adoption of international accounting standards. 
les risques financières, conduit à l'élaboration de ce document qui vise à approfondir sur le traitement comptable des actifs dérivés selon des règles différentes, se concentrant principalement sur la classification, lévaluation et la reconnaissance initial et intérieure de ces actifs.

Cet article analyse et compare le traitement comptable et financier des actifs dérivés sous différentes normes comptables, y compris : la norme locale du Chili selon les PCGA5, la norme américaine, sous le USGAAP et la norme européenne fondée sur les normes IFRS, afin de déterminer les différences significatives dans le traitement comptable des actifs dérivés. Pour atteindre cet objectif, nous avons procédé à la révision bibliographique des textes relatifs à la question de la valorisation des actifs dérivés, de l'administration des risques et des stratégies de couverture, ainsi comme à l'analyse des textes actualisés relatifs aux normes décrites, en obtenant comme résultats préliminaires quelques similitudes, comme celui dans lequel toutes les produits dérivés doivent se reconnaitre comme actifs et passifs, dans le bilan de la situation financière, initialement a la juste valeur et quelques différences comme les associées aux exigences formelles dans la comptabilité de couverture, la revalorisation des actifs dérivés implicites et les limites des couvertures, entre autres.

\section{Mots clés}

comptabilité, actifs dérivés, finances.

\section{Introducción}

\section{Descripción del problema}

Dada la globalización de los negocios y el nuevo entorno económico mundial, los distintos participantes (inversores, gobiernos, entidades

5 Avant l'adoption de règles comptable international 
multilaterales e internacionales del mercado de valores) han promovido he insistido en la eliminación de las barreras que impidan o comprometan la adecuada interpretación de los reportes financieros fundamentales en la gestión y decisiones gerenciales. De ahí, la importancia de contar con normas contables universales que permitan unificar criterios que faciliten las negociaciones entre distintas compañías a nivel mundial. Así, la creación de las IFRS ${ }^{6}$, constituyen un instrumento relevante a la hora de optar por la conversión, dado el deseo de unificar todas las normas contables y financieras alrededor del mundo.

Las nuevas directrices de las empresas chilenas y la participación de estas en nuevos mercados a nivel internacional, permiten calificar a Chile como una economía abierta que es afectada en forma importante por shocks de precios internacionales, incluyendo tipos de cambios, tasas de interés, precios de materias primas, entre otros. Por lo anterior, en el mundo financiero, se han desarrollado en forma significativa diversos tipos de instrumentos que permiten diversificar los riesgos a los que constantemente se ven enfrentadas las empresas y los intermediarios financieros, nos referimos a los: "activos derivados", instrumentos de gestión de riesgos.

Sin duda, evaluar la exposición a los riesgos de crédito, liquidez y cualquier otro tipo de riesgo de mercado a los cuales se enfrentan las empresas hoy en día, cobra vital importancia. Tener una eficiente política de gestión de activos derivados y de estrategias de coberturas, como así mismo, una revelación de la valorización, reconocimiento inicial, clasificación en los estados financieros básicos y la correcta aplicación de la normativa contable asociada a estos instrumentos, marcará la diferencia entre una compañía y otra a la hora de tomar decisiones financieras, más aún si se quieren incursionar nuevos mercados que deriven a la creación del valor de la empresa.

Por lo anterior, el presente documento pretende desarrollar un proyecto que analice y compare el tratamiento contable y financiero de los activos derivados bajo distintas normas contables, entre ellas: la local chilena

6 International Financial Accounting Standars (N. del E.) 
-PCGA- ${ }^{7}$, la norma americana, bajo los USGAAP $^{8}$ y la norma europea regulada por las IFRS.

\section{Objetivo general}

Determinar si existen diferencias significativas en el tratamiento contable de los activos derivados entre la norma contable chilena (PCGA), americana (USGAAP) y europea (IFRS).

\section{Objetivos específicos}

- Determinar las principales diferencias existentes entre las tres normas contables analizadas en el tratamiento de los derivados.

- Determinar las principales diferencias existentes entre las normas contables analizadas en el tratamiento de las coberturas de riesgo.

\section{Hipótesis de la investigación}

- Existen diferencias significativas entre la normativa contable de coberturas, aplicadas en Estados Unidos, Europa y Chile.

\section{Metodología de la investigación}

La metodología empleada en el desarrollo de este trabajo de investigación preliminar, se basó principalmente en la revisión bibliográfica de textos relacionados con el tema de valoración de activos derivados, administración del riesgo y estrategia de cobertura, así como el análisis de textos actualizados relacionados a las normas contables nacionales (PCGA), internacionales (IFRS) y norteamericana (USGAAP).

7 Principios de Contabilidad de General Aceptación (N. del E.)

8 Unites States General Accounting Accepted Principles (N. del E.) 
Dado que el tema en estudio abarcó asuntos contables y financieros más contemporáneos y de aplicación al mercado chileno, fue de vital importancia la revisión de artículos de revistas y documentos de trabajos publicados por instituciones académicas y públicas, como los documentos de trabajo del Banco Central de Chile o de la S.V.S. ${ }^{9}$ El análisis bibliográfico, nos permitió obtener importante información respecto de los instrumentos de derivados y su utilización por parte de los mercados financieros a nivel nacional y mundial, destacando a Chile, Estados Unidos y Europa con sus respectivas normas contables.

\section{Resultados de la Investigación - Parte I Generalidades de los instrumentos derivados}

\section{Revisión de la literatura sobre instrumentos derivados}

\section{1-1 Instrumentos derivados y sistemas financieros}

Diversos estudios empíricos han puesto en evidencia una relación positiva entre el funcionamiento y desarrollo de los mercados financieros con el crecimiento económico de largo plazo (Levine, 2005). Dentro de las funciones en las cuales el sector financiero puede contribuir al crecimiento económico, se encuentra aquella relacionada con permitir la transacción, diversificación y administración del riesgo.

En relación a lo anteriormente señalado, es posible argumentar que los mercados de derivados a través de los instrumentos de derivados, contribuyen en gran medida al cumplimiento de la función del sector financiero, ya que son instrumentos cuyo principal objetivo es el manejo de riesgos.

Sin lugar a dudas, la crisis financiera subprime desatada a mediados del 2008 en Estados Unidos, ha provocado que los instrumentos derivados estuviesen en el centro de las críticas y fuesen señalados como unos de los principales culpables en el desenlace de esta, debido a su rol preponderante

9 Superintendencia de Valores y Seguros (N. del E.) 
en todo el esquema que desencadenó el colapso financiero y su posterior propagación a nivel mundial. Sin embargo, un informe preparado por el Deutsche Borse Group (2009) afirma que los instrumentos derivados no causaron la crisis financiera, más aún, el mencionado informe señala que un conjunto apreciable de áreas de los mercados de derivados han continuado desempeñando bien su rol como instrumento de inversión y de protección del riesgo de mercado.

El mencionado estudio también señala que la realización de transacciones bursátiles de derivados y la liquidación a través de Entidades de Contraparte Central (CCPs) de derivados estandarizados transados ya sea OTC (Over the counter) o en el mercado bursátil, han constituido un elemento estabilizador y de resiliencia de mercado, principalmente en tres aspectos: (a) las CCPs efectivamente han cumplido un rol mitigador y de administrador de riesgos, (b) las CCPs han aportado a proveer de transparencia en las posiciones de riesgos, y (c) la liquidez y actividad del mercado de derivados se mantuvo durante y después de la crisis.

Por otra parte, Instefjord (2003) investiga si la ingeniería financiera de los derivados de crédito genera que los bancos se expongan aún más al riesgo de crédito. El autor identifica dos efectos asociados al surgimiento de estos derivados, señalando por una parte que estos instrumentos permiten una mejor distribución de riesgos, pero por otro lado pueden incentivar la toma de mayores niveles de riesgo de crédito.

En este mismo sentido, Kumar (2005) señala que el uso de los derivados de crédito ha facilitado la distribución del riesgo crediticio entre un más amplio grupo de inversionistas, lo cual tiene un efecto positivo sobre la estabilidad financiera. A este respecto, la práctica común por parte de los bancos ha sido mantener el riesgo de crédito en sus EEFF (Estados Financieros) en la forma de provisiones pro-cíclicas. En las últimas décadas, y previo a la crisis financiera subprime, los bancos crecientemente comenzaron a transferir sus exposiciones crediticias a terceros por medio de los mercados financieros. 
Por otra parte, Zigrand (2009) indica que los derivados poseen dos aspectos claves para la estabilidad financiera, las presencia de riesgos endógenos y la existencia de interconexiones del mercado. En relación al riesgo endógeno, este captura los espirales de precios y endeudamientos creados por decisiones de los agentes de mercado y con respecto a la interconexión del mercado, señala que normalmente el grado de interrelación de un sistema financiero se mueve en conjunto con mayores niveles de endeudamiento entre los bancos, en periodos de boom, lo que induce a una ampliación de los balances y a una expansión del riesgo sistémico en periodos de auge económico.

\subsection{Inicios de los derivados en Chile}

Estos instrumentos fueron lanzados en el mercado financiero local en 1992 y 1994, siendo transados principalmente entre instituciones financieras y entre estas y grandes compañías.

Se dice que las principales causas del escaso desarrollo del mercado doméstico de derivados son la iliquidez y la poca profundidad del mismo sistema financiero chileno. Precisamente, respecto de la introducción de los derivados en tasas de interés y derivados en instrumentos de renta fija en agosto de 1999 y septiembre de 2000, respectivamente. Paralelamente, se han introducido otras medidas que apuntan a la ampliación de las alternativas de inversión y a un aumento de la actividad bursátil. En efecto, en marzo de 1999 la S.V.S, autorizó el uso de las ventas cortas de acciones.

A la fecha, no obstante, estas han tenido escaso éxito. Ello se debería, primero, a que no cuentan con franquicia tributaria y, segundo, a que las AFPs ${ }^{10}$ no están autorizadas a realizar estas operaciones en circunstancias de que estas podrían ser los principales prestamistas de acciones dado el stock que mantienen. Es por ello que, posiblemente, en un futuro no muy lejano las empresas Telefónica CTC, Enersis y Endesa dejen de cotizar sus

10 Administradoras de Fondos Pensionales (N. del E) 
acciones en la rueda local. En conjunto, estas representan el $36 \%$ de la capitalización bursátil del IPSA (índice de Precios Selectivo de Acciones). De concretarse tal salida, la bolsa doméstica perdería gran liquidez y, en opinión de algunos, podría eventualmente desaparecer.

No obstante, a la fecha, la participación de las empresas locales medianas y pequeñas ha sido escasa en este mercado. Ello se debería al alto costo de su financiamiento y a un desconocimiento de cómo operan los instrumentos de cobertura. Sin embargo, dado que el riesgo cambiario es un tema clave para el comercio exterior, es de esperar que el uso de los instrumentos de cobertura de tipo de cambio abarque a un sector más amplio de la economía chilena a futuro.

Actualmente los productos derivados en moneda extranjera son los que gozan de mayor aceptación en el mercado local y son ampliamente utilizados por instituciones financieras y empresas del sector real. Antes de la crisis asiática en 1997, las empresas no le daban gran importancia a las fluctuaciones en el dólar, y en las paridades cambiarias, por lo que no adoptaban medidas de protección frente a estos riesgos financieros. Hoy en día, la gran mayoría de las empresas reconocen esta amenaza y son muchas las que se han visto afectadas por movimientos adversos en las variables mencionadas. En efecto, las fluctuaciones que se producen pueden ser determinantes en un negocio, transformando utilidades en pérdidas, como resultado exclusivo del efecto de la variabilidad de las paridades financieras.

En el caso de los bancos, los instrumentos permiten, por ejemplo, obtener financiamiento en dólares mucho más barato que pidiendo un crédito directamente a un banco extranjero, ya que este se encarece por el impuesto, el encaje, las primas, el premio por riesgo país, etc. También la utilización de instrumentos de derivados en esta nación se debe al manejo cambiario en Chile, poniendo particular énfasis en el régimen de tipo de cambio flotante implementado en 1999.

Lo que al llevar a cabo estos instrumentos lograrían un sector financiero bien desarrollado que ofrece oportunidades de cobertura a las empresas 
reduciendo su vulnerabilidad a través de efectos sobre el balance contable; y un nivel bajo y decreciente de traspaso desde el tipo de cambio a los precios. Estos elementos contribuyen a reducir los costos del régimen flotante, reduciendo la amenaza implícita en términos financieros y de inestabilidad de precios, y por lo mismo evitando el miedo a flotar. Además, provee suficiente credibilidad al actual sistema cambiario, y refuerza el compromiso con dejar las intervenciones para muy contadas ocasiones.

El desarrollo de un mercado de coberturas de la paridad es positivo pues permite a muchas empresas protegerse de las fluctuaciones del tipo de cambio. Por medio de estas operaciones, las empresas pueden ir fijando niveles de tipo de cambio para sus flujos futuros y así limitar la incertidumbre y la volatilidad de esperar hasta el pago de la transacción real, dedicándose así a su negocio principal.

\subsection{Principales derivados transados en Chile}

En nuestro mercado destacan los forward con un $82.4 \%$, seguido por los $f x$ swaps y cross currency con participación del $15.9 \%$ y $1.7 \%$, respectivamente. Por otra parte, el dólar es la divisa con mayor participación en el mercado de derivados, ya que está presente en el $99 \%$ de las transacciones ya sean peso-dólar (95\%), dólar-euro (2\%) y dólar-yen (2\%).

El mercado de derivados más importante en Chile es el contrato a plazo (forward) sobre moneda extranjera. Las contrapartes pueden acordar el uso de colateral para garantizar que la contraparte cumpla el contrato. Puede ocurrir que la entrega del subyacente sea innecesaria, cara, inconveniente o sujeta a impuestos o controles de capital; para evitar esto, los participantes de mercado pueden elegir transar forwards de moneda que se liquidan en dinero en una moneda; estos se llaman forwards sin despacho o entrega física.

Esta última es la modalidad de liquidación que se emplea en Chile en todos los contratos de derivados; por supuesto, los contratos locales se compensan en pesos (teniendo como referencia el tipo de cambio observado). 
Otro contrato relevante es el swap inter-monedas, en el que una corriente de pagos en pesos chilenos o en unidades de fomento se intercambian por una corriente de pagos en dólares; la tasa de interés que determina los pagos puede ser fija o variable, aunque usualmente los pagos en UF son a tasa fija. También se ofrecen en el mercado swaps peso-dólar, peso-euro y peso-yen.

En lo que se refiere a la madurez de los contratos, la gran mayoría de los ellos (forward y swaps) son a menos de un año, en el caso de forwards 73 $\%$ de los contratos vence entre 7 días y 1 año; y en el caso de los swaps 78 $\%$ de los contratos vence en el mismo plazo. Un $4 \%$ de los swaps tiene vencimientos mayores a 1 año, y solo un $1 \%$ de los forwards vence en más de un año. Esto sugiere que los plazos de los derivados tienden a ser cortos, no existiendo según estos antecedentes liquidez para plazos mayores a un año.

El mercado de forwards en Chile comenzó a popularizarse desde el momento en que el Banco Central eliminó la banda del precio sobre el dólar. Desde entonces hasta hoy, existe una gran profundidad en el mercado, en comparación con otros productos financieros. Según estadísticas publicadas por el $\mathrm{BCCh}^{11}$, en nuestro mercado se realizan operaciones de contratos a plazo y permutas financieras sobre monedas, los que en el mercado formal alcanzan en conjunto a unos 600 millones de USD por día. Como referencia, esta cifra representa poco menos de la mitad de las transacciones diarias del mercado spot de dólar. Adicionalmente hay un mercado (muy pequeño) de forwards de inflación. En la actualidad no se transan instrumentos derivados en bolsa, a pesar de existir la estructura legal y operativa necesaria.

Los instrumentos forward transados regularmente en Chile corresponden a contratos dólar/peso y dólar/unidad de fomento. Antes de la crisis asiática en 1997, las empresas no le daban gran importancia a las fluctuaciones en el dólar y en las paridades cambiarias, por lo que no adoptaban medidas de protección frente a estos riesgos financieros. Hoy en día, la gran mayoría de las empresas reconocen esta amenaza y son muchas las que se han visto afectadas por movimientos adversos. En efecto, las fluctuaciones que se

11 Banco Central de Chile (N. Del E.) 
producen pueden ser determinantes en un negocio, transformando utilidades en pérdidas, como resultado exclusivo del efecto de la variabilidad de las paridades financieras.

Otros instrumentos derivados, tales como las opciones sobre acciones y los futuros sobre índices accionarios, introducidos en 1990, han tenido escaso éxito.

\section{Resultados de la Investigación - Parte II Valoración y reconocimiento contable de los derivados}

\section{Proceso de adopción IFRS en Chile}

La S.V.S. inició oficialmente el proceso de adopción de las IFRS en el 2006, y en el oficio circular 368, (16/10/06), anunció el proceso de preparación para la convergencia, la que traería un cambio de los PCGA, proceso que en dicha fecha fue abordado por más de 100 países.

En el 2009 fueron 198 las compañías que comenzaron a adoptar el sistema IFRS tanto en la modalidad Full (95), como Proforma (103). Las 198 empresas representaban un $31 \%$ del universo total de firmas que debían cambiar su sistema de auditoría. Mientras que el porcentaje de empresas que lo haría entre el 2010 y 2011 alcanzaría la cifra de un $69 \%$. Al 31 de diciembre del 2011 ya eran más de 500 compañías que habían adoptado IFRS.

\section{1 ¿Por qué adoptar las IFRS?}

La utilización de un mismo lenguaje contable para la comunicación entre los distintos operadores a nivel global, constituye prácticamente una obligación a la apertura de la economía nacional. 
Sin duda que esta adopción trae grandes beneficiarios, entre ellos a los inversionistas, accionistas, directores, trabajadores de las compañías locales y al mercado en general, los que han tenido que hacer un importante esfuerzo en entender la nueva modalidad de la información financiera.

Tal como se veía venir, o como muchos lo anticipaban, los cambios que aparecieron después de la implementación de los estándares internacionales se hicieron evidentes. Si tan solo hacemos mención a algunas empresas como por ejemplo LAN, que disminuyó su patrimonio en un $11,31 \%$, o Aguas Andinas que aumentó su patrimonio un 39,3\% durante el primer trimestre del año 2008; diríamos que los cambios hacia la nueva normativa realmente fueron y son importantes.

Para los expertos, el tema va más allá que simples ajustes. Para Alejandro Merajver, director de IFRS de Price Waterhouse Coopers Chile, los efectos de retasaciones, moneda funcional, corrección monetaria o valorizaciones de inversiones explican muchas de las bajas o alzas experimentadas en los estados financieros de las empresas.

En definitiva, la implementación de las IFRS en los estados financieros de las compañías chilenas provocó que se generaran las primeras diferencias. Así las primerizas y valientes empresas que adoptaron las nuevas normas en sus estados financieros, han podido palpar fidedignamente los impactos que con tanto ímpetu se habían visto en empresas europeas o simplemente en alguna charla de implementación hacia la nueva normativa.

\subsection{Cobertura, una medida de protección financiera y contable}

Dada la volatilidad que introdujo la adopción de la nueva norma en los estados financieros de las empresas chilenas, muchos negocios se vieron enfrentados a grandes cambios y riesgos financieros. Uno de los mejores mecanismos para asegurar la operación de los mercados son las coberturas 
financieras, ya que estas permiten a las empresas poder planear con seguridad diversas transacciones desarrolladas a lo largo del tiempo en que la empresa está en funcionamiento. A pesar de la principal ventaja de las coberturas, que es "proteger", hay que saber utilizarlas, de lo contrario la empresa se vería enfrentada a un riesgo mayor que incluso podría acabar con la organización.

Por lo anterior, antes de utilizarlas, lo primero que debe hacer la empresa es medir el nivel de exposición al riesgo, es decir, debe analizarse qué parte de la operación debe cubrirse y establecer el monto específico. Estos mecanismos otorgan a los empresarios una alternativa para diversificar y administrar riesgos de los recursos económicos, pues permiten una planeación futura de los precios a los cuales se deberán cubrir los compromisos. Uno de los mercados que ofrece una serie de instrumentos creados para disminuir el riesgo generado por las constantes fluctuaciones de los precios internacionales que afectan a las compañías, es el mercado de derivados financieros.

El objetivo principal de los instrumentos financieros es distribuir el riesgo, al cual se enfrentan muchas empresas hoy en día. Movimientos inesperados en variables financieras como precios, tasas, índices, monedas, entre otros, como así mismo asegurar estabilidad en flujos de cajas, son algunos factores que llevan a las empresas, en cualquier parte del mundo, a acudir a un instrumento financiero. Sin embargo, para que una empresa pueda utilizarlos sin incurrir en la especulación, estas operaciones deben estar registradas conforme a una ley.

Hay que destacar un punto importante en el uso de las coberturas, el cual se relaciona con la complejidad en el manejo de estos instrumentos, ya que exige el conocimiento de sus características, de cómo pueden ser usados, de sus implicaciones, la forma de registrarlos contablemente, entre otras, de manera que puedan ser utilizados como herramientas para aumentar la rentabilidad de las empresas y disminuir sus riesgos. 


\subsubsection{Coberturas en Estados Unidos}

\section{Contexto general}

Una de las características sobresalientes de las coberturas, es el gran crecimiento que han experimentado, no solo el volumen de contratación o la negociación de estos en los mercados financieros, sino que también la complejidad de estos productos ha experimentado un impresionante aumento. Lo anterior ha provocado que la compra y venta de derivados haya tomado vida propia y que las compañías financieras que no tenían para qué usar estos productos los están usando para especular, derivando así en una de las principales razones de la cual estriba el crecimiento de este tipo de transacciones. Sin duda que las apuestas por los precios futuros han generado una volatilidad dañina en los precios del petróleo, los alimentos y otros sectores, así lo demuestra por ejemplo el dinero invertido en importar alimentos por parte de los países desarrollados, el cual aumentó de unos 190.000 a 254.000 millones de dólares entre 2006 y 2007, como consecuencia, principalmente, de la especulación con derivados de productos agrícolas y el petróleo.

\subsubsection{Rol de la FASB en temas de cobertura}

A lo largo de los años, la FASB (Financial Accounting Stándards Board) ha tenido dificultades para mantenerse al paso con las innovaciones financieras que utilizan las compañías para administrar o protegerse de la exposición a los riesgos resultantes de los repentinos cambios de los mercados financieros globales. Aunque unos pocos pronunciamientos han proporcionado reglas sobre la contabilidad de los derivados, esa orientación es algo fragmentada y a menudo resulta inconsistente e incompleta, porque solo unos pocos de los instrumentos financieros usados, hoy están cubiertos específicamente.

Después de varios años (1998) FASB emitió la declaración No. 133, (FAS 133): Contabilidad para los Instrumentos Derivados y Actividades de Cobertura. Esta norma establece las pautas contables y de información de los instrumentos derivados, incluyendo ciertos instrumentos derivados incorporados en otros contratos y para actividades de cobertura. Aunque 
la FAS 133, considera muchas de las actuales preocupaciones contables y acomoda muchas estrategias de cobertura, el nuevo enfoque es excesivamente complejo en su aplicación y no es necesariamente representativo de la forma en que es administrado el negocio.

\subsubsection{2 ¿Qué dice la FAS 133?}

La FAS 133, establece las normas contables y de información de los instrumentos derivados, incluyendo ciertos instrumentos derivados incorporados en otros contratos (conjuntamente denominados como derivados) y para actividades de cobertura. Se requiere que una entidad reconozca todos los derivados ya sea como activos o pasivos en el estado de situación financiera a su valor razonable, como así mismo, su medición. Si se cumplen ciertas condiciones, un derivado puede ser designado específicamente como:

a. Una cobertura de la exposición a los cambios en el valor razonable de un activo o pasivo reconocido o de un compromiso firme no registrado.

b. Una cobertura de la exposición al efectivo variable en las corrientes de una transacción prevista.

c. Una cobertura de la exposición en moneda extranjera de una inversión neta en un negocio en el extranjero, un compromiso firme no registrado, un activo financiero disponible para la seguridad-venta, o una transacción prevista en moneda extranjera emitidos. (FASB, 1997, pág. 26-27)

\section{¿Qué pasa posteriormente con los cambios en el valor razonable?}

La contabilización de los cambios en el valor razonable (VR) de un derivado (es decir, ganancias y pérdidas) depende del uso previsto de los derivados y la designación resultante, así según la FAS 33

1. "For a derivative designated as hedging the exposure to changes in the fair value of a recognized asset or liability or a firm commitment (referred to as a fair value hedge), the gain or loss is recognized in earnings in the period of change together with the offsetting loss or gain on the hedged item attributable 
to the risk Designado como una cobertura del VR, la ganancia o pérdida se reconoce en los ingresos en el periodo de cambio junto con el compensar la pérdida o ganancia de la partida cubierta atribuible al riesgo cubierto.

2. For a derivative designated as hedging the exposure to variable cash flows of a forecasted transaction (referred to as a cash flow hedge), the effective portion of the derivative's gain or loss is initially reported as a component of other comprehensive income (outside earnings) and subsequently reclassified into earnings when the forecasted transaction affects earnings.Designado como cobertura de flujos de efectivo, la parte eficaz de obtener la derivada o la pérdida que sea reportada inicialmente como un componente de otro resultado global (fuera de las ganancias), y posteriormente reclasificadas en las ganancias cuando la transacción prevista afecta a las ganancias. La parte ineficaz de la ganancia 0 pérdida de datos se expresan en las ganancias de inmediato.

3. For a derivative designated as hedging the foreign currency exposure of a net investment in a foreign operation, the gain or loss is reported in other comprehensive income (outside earnings) as part of the cumulative translation adjustment. Designado como cobertura del riesgo de moneda extranjera de una inversión neta en un negocio en el extranjero, la ganancia o pérdida se informa en otro resultado global (fuera de los ingresos) como parte del ajuste por conversión.

4. For a derivative not designated as a hedging instrument, the gain or loss is recognized in earnings in the periodPor un derivado no designado como instrumentos de cobertura, la ganancia o pérdida se reconoce en los ingresos en el periodo de cambio"12 (págs. 29-30).

\subsubsection{Coberturas en Europa}

El entorno donde básicamente se desarrolla el tema de los derivados es en Europa, sin embargo el origen de estos instrumentos y de los mercados de futuros y opciones se encuentra en la ciudad de Chicago, la que puede considerarse como el centro más importante en lo que a productos de derivados se refiere, y es aproximadamente diez años después de su creación

12 Nota: si una relación de cobertura no es retrospectiva, la cobertura se termina, y la ganancia diferida o pérdida del derivado se reconoce en las ganancias agregando una nota a los estados financieros. 
en Estados Unidos, en la década de los ochenta, que los contratos de futuros y opciones financieros llegan al viejo continente.

\subsubsection{La regulación de Activos Derivados en Europa}

La NIC 39 sobre instrumentos financieros, reconocimiento y valoración, entró en vigencia el 1 de enero de 2001, habiendo sido aprobada por el IASC (International Accounting Standards Committee) en diciembre de 1998. A nivel europeo, la NIC 39 es recogida por el reglamento (CE) número 2086/2004 de la comisión del 19 de noviembre de 2004. Esta norma está basada en la FAS 133, relativa a la contabilidad de instrumentos derivados y actividades de cobertura, publicado en junio de 1998 y con efectividad desde junio de 1999, muchos de sus apartados quedan prácticamente reproducidos en la NIC, manteniendo el mismo esquema.

\subsubsection{2 ¿Qué es un derivado según la IAS?}

Según IAS 39 "Instrumentos financieros: reconocimiento y valoración", un derivado es un instrumento financiero que tiene las siguientes características:

a. Valor que cambia en respuesta a los cambios en una tasa de interés, de un precio de acciones, precios de mercancías, etc.; o de una variable similar que a menudo se denomina subyacente.

b. Que requiere, al principio, una inversión neta muy pequeña o nula, respecto a otro tipo de contratos.

c. Su liquidación se hará en una fecha futura.

\subsubsection{3 ¿Cómo es la valoración inicial de los activos derivados, según NIC 39?}

Todos los productos derivados deben reconocerse como activos y pasivos en el balance de situación financiera, inicialmente a su costo que será el VR de la contraprestación que se haya dado o que se haya recibido a cambio de los mismos. 
El VR normalmente se determina a través del precio de la transacción o de precios de mercado. Si estos precios de mercado no pueden determinarse fiablemente, el VR se estimará como la suma de los futuros pagos o cobros, descontados, utilizando las tasas de interés del mercado para instrumentos similares para un emisor con la misma calificación crediticia.

\subsubsection{Valoraciones posteriores}

La valoración posterior de los activos y pasivos financieros corresponderá al VR de los mismos, mientras que los cambios de ese valor serán registrados en el resultado.

\subsubsection{Tipos de cobertura según IAS}

1. Cobertura del valor razonable (faire value hedge): es una cobertura de la exposición a los cambios en el VR de activos o pasivos previamente reconocidos en el balance $o$ de compromisos en firme ${ }^{13}$ no reconocidos, $\mathrm{o}$ bien una porción identificada de dichos activos, pasivos o compromisos en firme, que sea atribuible a un riesgo particular y que pueda afectar al resultado del ejercicio.

2. Cobertura de los flujos de efectivo (cash flor hedge): es una cobertura de la exposición a la variación de los flujos de efectivo que se atribuye a un riesgo particular asociado con un activo o pasivo previamente reconocido, o a una transacción prevista altamente probable y que pueda afectar al resultado.

"La cobertura del riesgo de tipo de cambio de un compromiso en firme puede ser contabilizada como una cobertura del VR o una cobertura de flujos de efectivo" (IASC, 2001, p. 34).

3. Cobertura de la inversión neta en un negocio en el extranjero: tal como se define en la NIC 21 (efectos de las variaciones en los tipos de cambio de la moneda extranjera). Por lo tanto, afecta específicamente a estados consolida-

13 Compromiso en firme: es un acuerdo obligatorio para intercambiar una determinada cantidad de recursos a un precio determinado, en una fecha o fechas futuras específicas (NIC 39). 
dos resultantes de las eliminaciones procedentes de entidades consolidadas relacionadas con diferencias de cambio surgidas en ese proceso y cubiertas.

\subsubsection{Tratamiento contable de los instrumentos derivados}

\subsection{Tratamiento contable de las coberturas sobre el valor razonable}

Si una cobertura del VR cumple durante el ejercicio con los requisitos establecidos para la contabilidad de coberturas, se contabilizará de la siguiente manera:

a. La ganancia o pérdida que resulte de la reexpresión contable, debe ser reconocida de forma inmediata en la ganancia o pérdida neta del periodo.

b. La ganancia o pérdida atribuible al riesgo cubierto del elemento protegido debe reconocerse también en resultados modificándose el valor contable del mismo. El resto de la ganancia o pérdida de este elemento no atribuible al riesgo cubierto se registra según las normas que le sean aplicables.

El ajuste que se haya realizado en el valor en libros de un instrumento financiero que produzca intereses, debe ser objeto de amortización o imputación a la ganancia o a la pérdida neta de la empresa. El importe del ajuste debe quedar totalmente amortizado o imputado en el momento del vencimiento.

La valoración de la inefectividad en la cobertura debe ser consistente con la estrategia de gestión de riesgo declarada por la empresa y el método de cálculo de la efectividad documentado al inicio de la relación de cobertura.

Una empresa cesará de aplicar la contabilidad de cobertura cuando:

a. El derivado expire, sea vendido o ejercido.

b. La cobertura ya no cumple los criterios para calificarla así contablemente.

La empresa puede elegir entre designar para el futuro una nueva relación de cobertura con otro derivado, o con otro elemento a cubrir siempre que la nueva relación cumpla los criterios exigidos. 
2.2.2.6.2 Tratamiento contable de las coberturas sobre los Flujos de Efectivo (FE)

Una cobertura sobre los FE se contabilizará si cumple con las siguientes condiciones:

a. La porción de la pérdida o ganancia que se haya determinado como una cobertura eficaz debe ser reconocida directamente en el patrimonio neto, a través del estado de variación en el patrimonio neto.

b. La porción determinada como ineficaz debe ser reconocida en el resultado.

Más específicamente, una cobertura del FE se contabilizará de la siguiente manera:

a. El componente separado de patrimonio neto asociado con la partida cubierta se ajustará para que sea igual (en términos absolutos) al menor valor de:

1. El resultado acumulado del instrumento de cobertura desde el inicio de la cobertura.

2. El cambio acumulado en el valor razonable (valor actual) de los FE futuros esperados de la partida cubierta desde el inicio de la cobertura.

2.2.2.6.3 Tratamiento contable de las coberturas sobre la inversión neta en moneda extranjera

Estas coberturas se contabilizarán de manera similar a las coberturas de FE:

a. La parte de la ganancia o pérdida que se califique como cobertura eficaz, debe ser reconocida directamente en el patrimonio neto, a través del estado de cambios en el patrimonio neto.

b. La parte calificada como ineficaz se reconocerá directamente en el resultado.

La pérdida o ganancia del instrumento de cobertura relativa a la parte de cobertura que ha sido reconocida directamente en el patrimonio neto 
se llevará al resultado en el momento de la venta o disposición por otra vía del negocio en el extranjero

\subsubsection{Coberturas en Chile - Boletín Técnico (B.T.) $N^{\circ} 57$}

El Boletín Técnico -BT-. se presenta antes de la entrada en vigencia de las IFRS. En Chile, la contabilización de instrumentos derivados, tales como los contratos de forward, futuros, swaps y opciones, ya sea tomados para efectos de cobertura o bien como alternativa de inversión temporal, está estipulada en el B.T. No 57 emitido por el Colegio de Contadores de Chile A.G., quien es el que clasifica a los contratos de derivados en:

\section{Contrato de cobertura (hedging)}

Son contratos tomados con el objeto de protegerse contra riesgos de variaciones de precio, tasas de interés, tipos de cambio, etc. A su vez, estos contratos se subdividen en:

\section{- Contratos de cobertura de partidas existentes}

Cuyos objetivos son proteger un riesgo al que están expuestas una o varias partidas existentes, ya sean activos o derechos o bien obligaciones o compromisos. Tanto en la contabilidad como en los EEFF, la partida protegida y el instrumento derivado deberán registrarse a su valor justo. La eventual diferencia existente entre el valor justo y el valor libros de la partida protegida, al inicio del contrato, se registrará de acuerdo al tratamiento habitual de esa partida.

\section{- Contratos de cobertura de transacciones esperadas}

Cuyos objetivos son proteger el riesgo del flujo de caja que se originará en una transacción que es probable se lleve a efecto a futuro (coberturas de flujos de caja). El instrumento de cobertura (derivado) deberá ser presentado a su valor justo y los cambios en dicho valor deberán ser reconocidos como resultado (ganancia o pérdida) no realizado. Al término del contrato, el resultado no realizado del instrumento derivado deberá reconocerse como un resultado realizado (ganancia o pérdida) por el uso de derivados, sin afectar el costo o precio de venta del activo adquirido o vendido en la transacción. 


\section{Contratos de inversión (non-hedging):}

Se suscriben con el objeto de obtener una utilidad u otro objetivo distinto al de cobertura expuestos anteriormente. En los estados financieros, el contrato de derivados deberá presentarse a su valor justo. Los cambios en este deberán ser reconocidos como resultado del periodo en que se originó dicho cambio.

En ningún caso el lanzamiento de una opción podrá ser considerado cobertura, por lo cual, el lanzador de la opción siempre deberá aplicar las normas correspondientes para los contratos de inversión, registrando, en consecuencia, las pérdidas que originará el ejercicio de la opción que haga la otra parte.

Tabla 1. Resumen con algunos de los principales hallazgos encontrados.

\begin{tabular}{|c|c|c|c|}
\hline Ítem & IAS 39 & FAS 133 & BT 57 \\
\hline $\begin{array}{l}\text { Definición de } \\
\text { "derivado". }\end{array}$ & $\begin{array}{l}\text { Instrumentos } \\
\text { financieros cuyo } \\
\text { valor cambia como } \\
\text { respuesta a un } \\
\text { índice específico } \\
\text { (por ejemplo, una } \\
\text { tasa de interés), } \\
\text { que no requiere } \\
\text { inversión o poca } \\
\text { inversión y que se } \\
\text { cancela en una fecha } \\
\text { futura. }\end{array}$ & $\begin{array}{l}\text { Se establecen } \\
\text { requerimientos } \\
\text { similares, } \\
\text { exceptuando que } \\
\text { en los términos } \\
\text { del contrato de } \\
\text { derivado debe } \\
\text { requerirse o } \\
\text { permitirse una } \\
\text { liquidación neta. }\end{array}$ & $\begin{array}{l}\text { Definición similar a } \\
\text { las otras dos normas } \\
\text {, señalando el } \\
\text { derecho u obligación } \\
\text { que proporciona al } \\
\text { tenedor de participar } \\
\text { total o parcialmente } \\
\text { del cambio en el valor } \\
\text { de un ítem específico, } \\
\text { sin necesidad que } \\
\text { este sea propietario } \\
\text { de ese ítem o requiera } \\
\text { entregarlo o recibirlo. }\end{array}$ \\
\hline $\begin{array}{l}\text { Medición } \\
\text { inicial. }\end{array}$ & $\begin{array}{l}\text { Se miden } \\
\text { inicialmente al valor } \\
\text { razonable en la } \\
\text { fecha de compra. }\end{array}$ & Similar a la IAS. & $\begin{array}{l}\text { Similar a la IAS y a } \\
\text { la FAS. }\end{array}$ \\
\hline
\end{tabular}




\begin{tabular}{|c|c|c|c|}
\hline Ítem & IAS 39 & FAS 133 & BT 57 \\
\hline $\begin{array}{l}\text { Medición } \\
\text { posterior. }\end{array}$ & $\begin{array}{l}\text { A su valor } \\
\text { razonable, } \\
\text { independientemente } \\
\text { de que alguna } \\
\text { relación de } \\
\text { cobertura pueda } \\
\text { existir. Los cambios } \\
\text { en el valor razonable } \\
\text { se reconocen en el } \\
\text { estado de resultado. }\end{array}$ & Similar a la IAS. & $\begin{array}{l}\text { Similar a la IAS y a } \\
\text { la FAS. }\end{array}$ \\
\hline $\begin{array}{l}\text { Criterios para } \\
\text { utilizar la } \\
\text { contabilidad } \\
\text { de } \\
\text { coberturas. }\end{array}$ & $\begin{array}{l}\text { La contabilidad de } \\
\text { cobertura se permite } \\
\text { cuando una entidad } \\
\text { cumple con los } \\
\text { rigurosos criterios } \\
\text { relacionados con la } \\
\text { documentación y } \\
\text { la efectividad de la } \\
\text { cobertura. }\end{array}$ & Similar a la IAS. & $\begin{array}{l}\text { Similar a IAS y a la } \\
\text { FAS. }\end{array}$ \\
\hline $\begin{array}{l}\text { Tipos de } \\
\text { relaciones de } \\
\text { cobertura. }\end{array}$ & $\begin{array}{l}\text { - Coberturas a valor } \\
\text { razonable. } \\
\text { - Coberturas del } \\
\text { flujo de efectivo. } \\
\text { - Cobertura de } \\
\text { la inversión neta } \\
\text { en operaciones } \\
\text { extranjeras. }\end{array}$ & Similar a la IAS. & $\begin{array}{l}\text { Similar a la IAS y a } \\
\text { la FAS. }\end{array}$ \\
\hline $\begin{array}{l}\text { Medición de } \\
\text { instrumentos } \\
\text { financieros y } \\
\text { actividades } \\
\text { de cobertura. }\end{array}$ & $\begin{array}{l}\text { Se reconocen los } \\
\text { cambios en el valor } \\
\text { razonable en el } \\
\text { estado de resultado, } \\
\text { con excepción de } \\
\text { las coberturas de } \\
\text { flujos de efectivo } \\
\text { que sean efectivas, } \\
\text { cuyos cambios } \\
\text { son diferidos en } \\
\text { el patrimonio } \\
\text { hasta que se } \\
\text { reconoce el efecto } \\
\text { de la transacción } \\
\text { subyacente en el } \\
\text { estado de resultado. }\end{array}$ & Similar a la IAS. & $\begin{array}{l}\text { Se reconocen los } \\
\text { cambios en el valor } \\
\text { razonable en el estado } \\
\text { de resultado. En las } \\
\text { coberturas de flujo de } \\
\text { caja los cambios en } \\
\text { dicho valor deberán } \\
\text { ser reconocidos como } \\
\text { resultado (ganancia o } \\
\text { pérdida) no realizado, } \\
\text { y al término del } \\
\text { contrato deberá } \\
\text { considerarse como un } \\
\text { resultado realizado. }\end{array}$ \\
\hline
\end{tabular}




\begin{tabular}{|c|c|c|c|}
\hline Ítem & IAS 39 & FAS 133 & BT 57 \\
\hline $\begin{array}{l}\text { Medición } \\
\text { de las } \\
\text { coberturas } \\
\text { de las } \\
\text { inversiones } \\
\text { en entidades } \\
\text { extranjeras } \\
\text { (IEE). }\end{array}$ & $\begin{array}{l}\text { Las ganancias/ } \\
\text { pérdidas en } \\
\text { coberturas de las } \\
\text { IEE se reconocen } \\
\text { en el patrimonio, } \\
\text { incluyendo las } \\
\text { coberturas no } \\
\text { efectivas que no } \\
\text { sean relacionadas } \\
\text { a instrumentos } \\
\text { derivados. }\end{array}$ & Similar a la IAS. & $\begin{array}{l}\text { Los cambios en } \\
\text { el valor razonable } \\
\text { deberán ser } \\
\text { reconocidos como } \\
\text { resultado del periodo } \\
\text { en que se originó } \\
\text { dicho cambio. }\end{array}$ \\
\hline $\begin{array}{l}\text { Fecha para } \\
\text { designar } \\
\text { cualquier } \\
\text { activo o } \\
\text { pasivo } \\
\text { financiero a } \\
\text { ser medido a } \\
\text { VR a través } \\
\text { de utilidad o } \\
\text { pérdida. }\end{array}$ & $\begin{array}{l}\text { Elección } \\
\text { solamente en el } \\
\text { reconocimiento } \\
\text { inicial. }\end{array}$ & $\begin{array}{l}\text { Elección en el } \\
\text { reconocimiento } \\
\text { inicial y también } \\
\text { en ciertas fechas } \\
\text { subsiguientes. }\end{array}$ & Similar a las FAS. \\
\hline $\begin{array}{l}\text { Límites de } \\
\text { cobertura. }\end{array}$ & $\begin{array}{l}\text { Se encuentran } \\
\text { dentro del rango de } \\
80 \% \text { a } 125 \% \text {. }\end{array}$ & $\begin{array}{l}\text { Asume la } \\
\text { efectividad } \\
\text { perfecta para } \\
\text { ciertas relaciones } \\
\text { de cobertura. }\end{array}$ & $\begin{array}{l}\text { Sobre el } 100 \% \\
\text { de riesgo a cubrir } \\
\text { considerando } \\
\text { el exceso como } \\
\text { inversión. }\end{array}$ \\
\hline $\begin{array}{l}\text { Revaloración } \\
\text { de } \\
\text { la condición } \\
\text { de } \\
\text { derivados } \\
\text { implícitos. }\end{array}$ & $\begin{array}{l}\text { No se permite } \\
\text { que las entidades } \\
\text { vuelvan a valorar si } \\
\text { se requiere que el } \\
\text { derivado implícito } \\
\text { sea separado, a } \\
\text { menos que haya } \\
\text { un cambio en } \\
\text { los términos que } \\
\text { modifiquen de } \\
\text { manera importante } \\
\text { los flujos de efectivo. }\end{array}$ & $\begin{array}{l}\text { Típicamente, las } \\
\text { entidades vuelven } \\
\text { a valorar si se } \\
\text { requiere que la } \\
\text { característica } \\
\text { implícita sea } \\
\text { separada al } \\
\text { menos al final de } \\
\text { cada periodo de } \\
\text { presentación de } \\
\text { reportes. }\end{array}$ & No especifica. \\
\hline
\end{tabular}

Fuente: elaboración propia. 


\section{Conclusiones}

Sin lugar a dudas a nivel mundial el interés por contratar productos derivados ha aumentado. Lo anterior, se debe especialmente a la existencia de un mercado con alta volatilidad de las divisas durante los últimos dos años, explicado principalmente por la inestabilidad en la eurozona, lo que repercute en que la demanda por coberturas cambiarias anote una fuerte expansión. Si consideramos, que en nuestro país, el volumen promedio diario de negocios de contratos a futuro, forwards, swaps y opciones en los dos primeros meses de este año se incrementaron en un $41,5 \%$ en comparación al mismo periodo del año anterior, diríamos que estamos frente a una estrategia de negocios que promete un futuro seguro en las finanzas y en los números contables ante los riesgos financieros.

¿Pero qué pasa con el tratamiento contable de estos contratos? A nivel mundial, existen normas que permiten facilitar el camino hacia la contabilización de estos activos, sin embargo muchas de ellas son específicas para cada país. En nuestro análisis, pudimos encontrar algunas similitudes y diferencias que se generan al momento de valorizar y reconocer instrumentos de derivados, ya sea en EE. UU, Europa o en Chile, entre las cuales destacamos aquella similitud que indica que todos los productos derivados deben reconocerse como activos y pasivos, en el balance de situación financiera, inicialmente a su valor razonable.

Por otra parte, entre las diferencias encontradas se destacan aquellas asociadas a los requerimientos formales en la contabilidad de coberturas, la revalorización de los activos derivados implícitos, los límites de las coberturas, entre otras, (ver tabla), lo que nos confirma la hipótesis planteada al inicio de nuestro trabajo. En síntesis, un mercado de coberturas en expansión genera una madurez en los inversionistas frente a una mayor oferta de productos financieros, evidenciando en las empresas una mayor preocupación respecto a tomar coberturas frente a riesgos financieros importantes, como así mismo genera la preocupación de querer aplicar la normativa contable acorde a cada movimiento de estos activos en los 
mercados, lo que conlleva a una constante especialización por parte del equipo contable de las empresas.

\section{Referencias}

Ahumada, L., y Selaive, J. (2007). Desarrollo del mercado de derivados cambiarios en Chile. Revista de Análisis Económico, 22(1), 35-58.

Budnevich, L. C., y Zurita, L. S. (2010). Diagnóstico, evaluación y propuesta de desarrollo del mercado de derivados en Chile. Documentos de trabajo, Santiago, Chile: Superintendencia de Valores y Seguros.

Cotach. (1997). Boletín Técnico No 57: contratos de derivados.

Deutsche Borse Group. (1998). The global derivatives market: A blueprint for market safety and financial accounting standard board. FAS 133 Contabilidad de cobertura.

Integrity. (2009). White Paper. London: IASC.

IASB. (1993). NIC 21. Efectos de las variaciones en los tipos de cambio de la moneda extranjera.

IASB. (2000). NIC 39. Instrumentos financieros: reconocimiento y valoración.

Kumar. (2005). Development of credit derivatives markets: Implications on monetary policy and financial stability of developing economies like India, mimeo. Department of Economics Mangalore University.

Mathematics and Statistics. (2005). School of Economics. London, England: Mimeo Birkbeck College.

Norvald, I. (2003). Risk and hedging: Do credit derivatives increase bank risk? Paper presentado en la conferencia: Derivatives in crisis: Safeguarding financial stability.

Ross, L. (2005). Finance and growth: Theory and evidence, Philippe Aghion \& Steven Durlauf (ed.), edition 1. Handbook of economic growth, 1(12), 865934, Elsiever.

Zigrand, J. (2009). An economist's view on derivatives and financial stability. Discurso presentado en el marco de la conferencia "Derivatives in crisis: Safeguarding financial stability”. London, England: London School of Economics, september 25. 